\title{
SHIP DETECTION USING SENTINEL-1 SAR DATA
}

\author{
Aayush Grover ${ }^{1, *}$, Shashi Kumar², Anil Kumar ${ }^{1}$ \\ ${ }^{1}$ School of Computer Science, University of Petroleum and Energy Studies, Dehradun, India - \\ aykgrover@gmail.com, anil.kumar@ddn.upes.ac.in \\ ${ }^{2}$ Photogrammetry \& Remote Sensing Department, Indian Institute of Remote Sensing, Indian Space Research Organization, Dehradun, \\ India - shashi@iirs.gov.in
}

\section{Commission V, SS: Atmosphere, Ocean, Weather and Climate}

KEY WORDS: Ship detection, Synthetic Aperture Radar (SAR), Sentinel-1, SUMO, marine objects, false alarms, sea, surveillance, land mask, threshold, identification.

\begin{abstract}
:
The Earth's surface is covered with $72 \%$ water. This fact alone emphasizes the importance of proper monitoring and regulation of maritime activities. This monitoring can be useful in an array of applications including illegal transitions, rescue operations, territory regulation among many other applications. In order to achieve the task of "Maritime Surveillance" or simply the marine object detection, we need a structured approach combined with a set of algorithms. The objective of this paper is to study an emerging open source toolSearch for Unidentified Maritime Objects (SUMO) developed for the detection of ships which work regardless of weather conditions and coverage limits. Based on the Synthetic Aperture Radar (SAR) data, this paper aims to process the satellite-borne data provided by the Sentinel-1 satellite. Proposed by the Joint Research Centre, SUMO is a pixel-based algorithm which follows a structured approach in order to identify marine objects and remove false alarms. It is observed that many of the false alarms are caused due to the presence of land. These are reduced by using the buffered coastlines referred to as land masks. A local threshold is calculated using the background clutter for the generation of false alarm rate and the pixels above this threshold are identified and clustered to form targets. A reliability value is computed for the elimination of azimuth ambiguities. Also, various attributes of the detected targets are calculated in order to give an accurate description of ships and its characteristics. With the SAR data being freely available due to the open data policy of the EU's Copernicus program, it has never been more viable to employ new methods for marine object detection and this paper explores this possibility by analyzing the results obtained. Specifically, the employed data consists of Sentinel-1 fine dual-pol acquisitions over the coastal regions of India.
\end{abstract}

\section{Introduction}

Marine reconnaissance is defined as the monitoring of human activities at sea. The surveillance is intended to support efforts related to security, safety, environmental and sustainability aspects (H Greidanus, Argentieri, Alvarez, Santamaria, \& Kourti, 2017). The surveillance over the water bodies, being one of the major transport routes is of paramount importance for any territory, which includes the sea congestion control, illegal transitions, and fishery management. In the earlier days, marine surveillance was controlled by patrol ships and tracking devices. The type of surveillance systems where the ships themselves report their location is referred to as cooperative or reporting systems. Automatic Identification System (AIS) and Vessel Detection System (VMS) are among the most commonly employed option. There is another type of surveillance which doesn't require cooperation on the side of vessel, known as the non-cooperative systems (Gao \& Parzen, 2011). These systems commonly use imaging sensors like cameras and radars located on a variety of platforms like ships, airplanes, satellites, etc. for marine objects detection. Ship detection with satellite enables detection of vessels which do not carry any tracking system on board such as smaller fishing ships and majorly the ships that are in the surveyed area illegally. Airborne and Satellite-borne data have enabled the monitoring of marine objects from a distant region, independent of ground circumstances (Juan, Lijie \&

\footnotetext{
* Corresponding Author
}

Xuelan 2009). Optical sensors and imaging radars are the most applied methods which collect data on a global scale for target detection and identification. Detection of maritime objects is an advanced application of satellite-borne SAR imagery. Sentinel-1 is the SAR satellite of the Copernicus programme managed by European Space Agency. Sentinel-1 satellite returns high resolution single-pol and dual-pol radar data worldwide on a routine basis. Some Sentinel-1 images over the seas and coasts are studied and two of them are discussed in this paper. The acquired data is then combined with an automated detection system for the identification of stationary and non-stationary objects present in the sea/ocean (Harm Greidanus et al., 2017). The open and free policy of European Union Copernicus has made the SAR imagery easily available on the net. Search for Unidentified Maritime Objects (SUMO) is an open source software, developed by the JRC (Joint Research Centre) for the purpose of tracking down marine objects using satellite imagery. The SUMO software executes the different sub-functions of ocean object detection in a single algorithm with the objective of finding ships in satellite images(Fernandez Arguedas et al., 2015). Being developed for the last 15 years, SUMO has been programmed to identify ships of all shapes and sizes and is compatible with almost every SAR equipped satellite data, including TerraSAR-X, RADARSAT-1,2, SENTINEL-1,2 etc. (Santamaria et al., 2017). In this paper, the algorithmic approach of marine objects' detection as well as the elimination of false alarms by SUMO is discussed while studying the real-time SAR data. SUMO has two modes of operation - semi automatic and fully automatic, increasing the ship detection capability and 
implementing a standard means of removing ambiguity. The estimation of targets' attributes enables the discrimination of various ambiguities like small islands, reefs, oil spills etc. The research paper aims at studying the SUMO algorithm and implementing it for results and discussions.

\section{Research Background: SAR and SUMO}

Synthetic Aperture Radar (SAR) is considered one of the best techniques for capturing radar images of Earth's surface with a massive amount of SAR equipped satellites already in the play. SAR images received from Sentinel -1 give an overview of the local background pixel stats. A SAR image is formed by the radar backscatter of different objects on the Earth's surface. The areas of water bodies such as sea, ocean, river etc. are depicted as darker pixels, whereas structures like buildings, bridges, islands appear brighter. The reason behind the bright pixels of man-made structures is the presence of metal corner reflectors and their perpendicular alignment with respect to the flight direction causing multiple reflections (McCandless, SEASAT Program Manager, \& Jackson, Chp-1). Large ships and small boats are depicted as clusters of bright pixels and appear to be a white spot against a darker, noisier background, which is a water body (sea). In simplified terminology, the constraint of ship recognition is merely the point target detection in the satellite radar imagery (Pichel, Clemente-Colon \& Wackerman- 2004). Moreover, an image obtained from a SAR equipped satellite is too big to fit on a computer screen and the only option is to scan the radar image visually for comparatively brighter spots, taking much time. Besides the detection of maritime objects, their attributes' extraction also becomes a significant part of the identification phase. The much needed automatic target detection in radar images has been implemented as an algorithm/software in SUMO. SUMO has been developed for the exploitation of SAR imagery for the detection of marine objects based on the pixel value intensities. SUMO is a ship detector tool designed as a Java software package. Fundamentals of the detection approach followed by SUMO are to find locally bright pixels in the water body (sea) and it makes use of only amplitude images. A ship can only be located by the algorithm when its pixel value is higher than the mean of background and noise, but the same situation might occur in the case of background pixel value that might accidentally attain similar high pixel value causing an ambiguity. A local threshold is computed to select bright pixel indicating the presence of ships in the sea. The flow of SUMO algorithm is discussed in the next section.

\section{SUMO: Methodology and Flow of Algorithm}

After imaging from the source data of SAR sensors, the goal is to load a land mask to remove the land area from the image. There are many objects on the surface like some small islands, harbor lighthouses, and other equipage which exhibit similar attributes to ships in the radar image and can give rise to false alarms. The existence of targets on the land area can significantly inflate the false alarm rate and therefore increment the workload of marine object detection, and decrement the rate of correct identification. To deal with these ambiguities on the ground, SUMO uses global coastline vector files. The land mask differentiates surface from the sea level. Also, the land masks used by SUMO are available with seaward buffering by various distances. Land mask with seaward buffering by some meters will be able to give you the

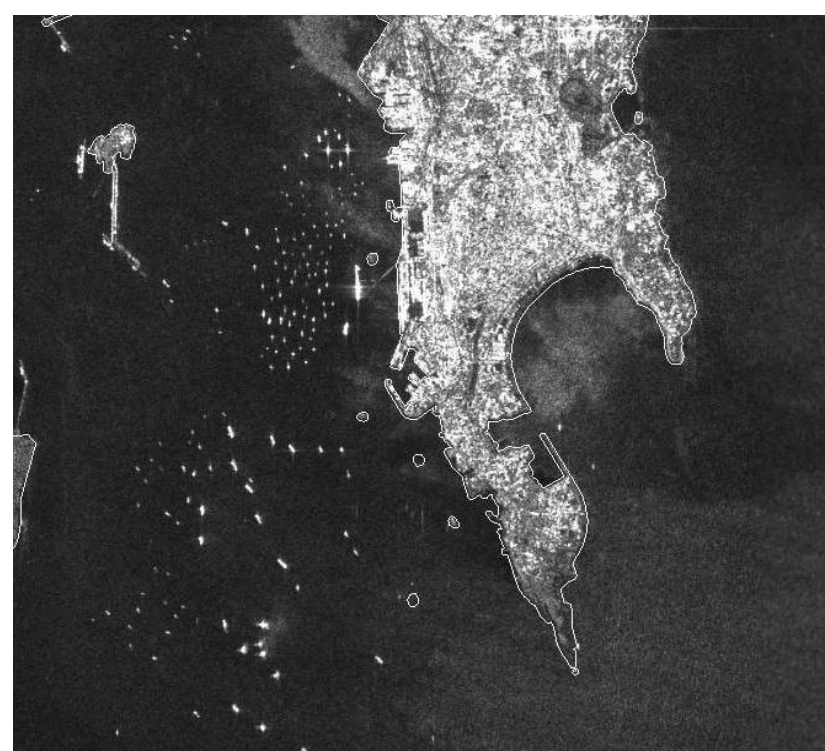

Fig 1. Jawaharlal Port, Mumbai (west coast of India). The white bright spots in the black background (sea) depict marine objects.

detection result of ships present at sea, but for the detection of those which are anchored off the shore, the user must select the land mask with minimum sea buffering to keep the coastline as close to the actual point of distinction (McCandless et al., n.d.). A buffered land mask also has the advantage that it is coarser, i.e. it needs fewer points so is faster to load and process. Next step is the estimation of the clutter background characteristics. SUMO implements the Kdistribution model to deal with the erratic scattering of radar backscatter over a ship, by assuming that the sea clutter complies with a K-distribution (Oliver \& Scitegh, 2004). While considering the sea clutter, the K-distribution model has three major parameters. One of them is PDF mean (denoted as $\mu$ ) and the other two are the PDF width measures. L denotes the ENL and $v$ measures the nonhomogenous nature of the backscatter over the sea. When the backscatter over the sea surface remains constant, $v$ tends to infinity means the $\mathrm{K}$-distribution limit is acting as a gamma curve and $v$ equal to one depicts the most inhomogeneous backscatter over the sea surface (Greidanus, H \& Clayton, P \& Indregard, Marte \& Staples 2004). The CFAR (amplitude) threshold- $\vartheta_{F A}$ can be computed by the integral:

$$
\int_{\vartheta_{\mathrm{FA}}}^{\infty}(\mathrm{f} \mathrm{k}(\mathrm{a}) \cdot \mathrm{da})=\text { PFA }
$$

with $\mathrm{f} \mathrm{k}$ (a) being the amplitude PDF of the assumed sea clutter (Kdistributed. The local background pixel parameters are computed using this distribution through small non-overlapping windows. Setting an overall threshold is not meaningful, so an adaptive threshold is set for each of the local windows. The local threshold is calculated according to the CFAR approach. Based on the local statistics, a Probability Density Function(PDF) of the background clutter is calculated for the estimation of the threshold value, above which any pixel value has a probability of being part of a ship (Vespe \& Greidanus 2012). SUMO is known as a CFAR detector because the resultant false alarm rate remains constant throughout a local window. The pixels brighter than the computed local threshold are identified which later form target ships. All the small and large groups of nearby detected pixels are clustered together into one 
detected target. In the case of multi-polarization images, the above steps are repeated for each of the polarization channels separately, and every detected pixel is taken in association ('logical or') over the channels. A target is considered detected if it has been identified above the threshold in at least one of the polarization channels, comprising of all the small and large sized marine objects. Specifically, while using the Sentinel-1 data (dual polarimetry here), the combination of cross-pol and $\mathrm{HH} / \mathrm{VV}$ yields the best possible results: cross-pol yields a high contrast between the target objects and the waterbody(Liu, 2015), but some marine objects have a low cross-pol RCS, and at far ranges, the cross-pol signal becomes weak; there, the HH/VV channel can still yield a good contrast (Olsen, Richard \& Hannevik, Tonje \& Eldhuset, Knut. 2004).

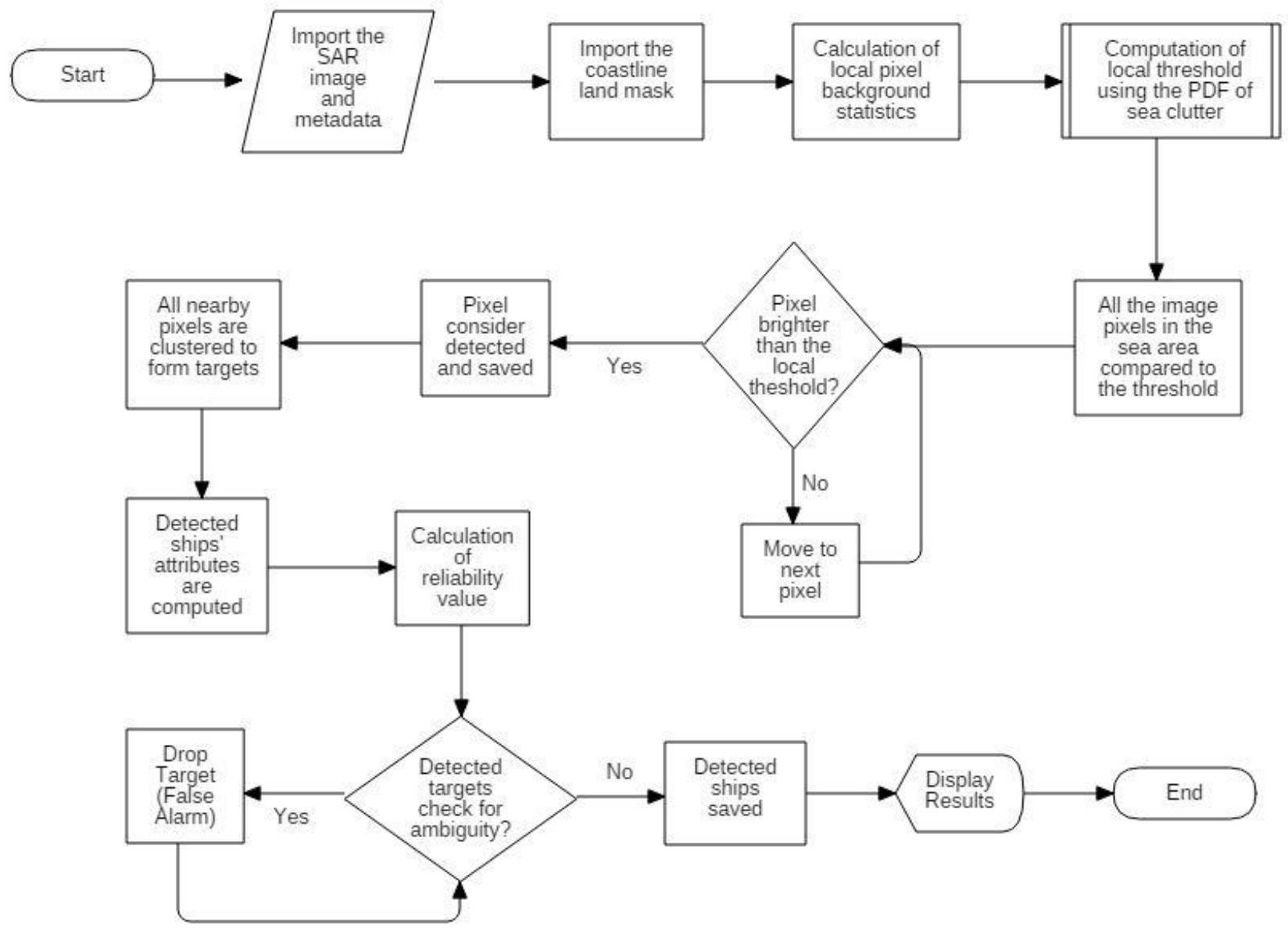

Figure 1. The flow diagram of ship detection and identification by SUMO

\subsection{Removing ambiguities:}

Some bright pixels may raise a false alarm and the discussed algorithm might take those pixel values in consideration and indicate the presence of ships even if they aren't present. After detecting ship targets, the next step is to remove the false alarm sources. The ambiguities may arise due to many reasons stated; the one to consider above all is that the objects on land are easily removed by the land mask, apart from this there is still a high probability of false alarms due to other factors (Wackerman, Friedman, Pichel \& Clemente-Colón 2001). Some prominent false alarm sources include small islands, off-shore construction sites, unrecognized azimuth ambiguities and range ambiguities from strong scatters on land, such as cities, certain mountain slopes that are not included in the land mask. Such false alarms tend to recur in the same position every time, so the same scenario is imaged again and again. The fixed orbit pattern of a satellite leads to repeated imaging of the same scene in the exact same geometry, as, e.g., exploited by interferometry. Another type of false alarms which arises in maritime SAR images are known as azimuth ambiguities. These are image artifacts, repetitions of targets at a much lower level at fixed distances in azimuth. In most cases, the azimuth ambiguities of a target are below the clutter level, but for strong targets and low clutter level they may be visible and could be detected (Fernandez Arguedas et al., 2015). Bright targets on land near the coast may produce azimuth ambiguities on the sea, this generally happens near ports. SUMO checks if there is a brighter target than the previous at the known azimuth ambiguity distance on either side of a detected target, and if so, the detection is flagged and raised as a false alarm. Also, a reliability value is computed for each of the detected ship targets based on the 
significance and the attributes of the ships for the elimination of azimuth ambiguities (Vespe, Michele and Harm, 2012). Another type of false alarm is the range ambiguities. But due to the large offsets, distance puts the possible range ambiguity source outside of the image, precluding any verification. Thus, SUMO doesn't attempt for any check on range ambiguities. However, for a series of repeat-pass images, range ambiguities can often be identified not treated. Especially for Sentinel-1, which acquires imagery over the same designated areas in each overpass, many repeat-pass images are available. This makes it possible to flag recurrent targets and indicate them as false alarms in the post-processing phase.
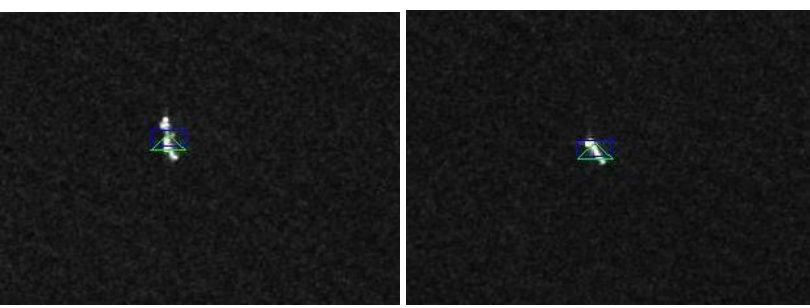

Fig.2 Zoom-ins of detected ship targets. A detected target is considered a ship taking association with detections over all the polarization channels.

\subsection{Attributes Extraction}

The various attributes of the detected targets like length, width, heading direction, etc. are calculated in order to give an accurate description of ships and their characteristics. The size estimation is complex in SAR images due to the limited resolution and because of SAR image distortions and the artifacts that blur the outline of moving targets(Vachon \& Wolfe, 2008). SUMO estimates the heading direction and the size of the ships by treating the detected pixels (before clustering) as points on a Cartesian grid (x,y) and fitting minimum squares line to this point cluster. Ship's heading direction is estimated by considering the orientation of the fitted line with respect to the range direction and the size is estimated by measuring the span of bordering pixels. Length is calculated by the distance between the extremes along the line while the distance perpendicular is taken as the width. These attributes can be saved in an output XML file with various attributes in tags of each detected target.

The other attributes which can be extracted from the output results of SUMO detection are:-

- $\quad$ Pixel number and intensity

- Geographic location in longitude \& latitude

- Number of detected pixels and the maximum pixel value

- Reliability value

- Radar Cross-Section (RCS)

\subsection{Study Definition}

\section{Study Definition and Dataset}

The area of interest (AoI) of the study is the Coastal areas of India (including the Arabian Sea and the Bay of Bengal). The study period is almost two months, from May 2018 to June 2018. Figure 3 maps the AoI.

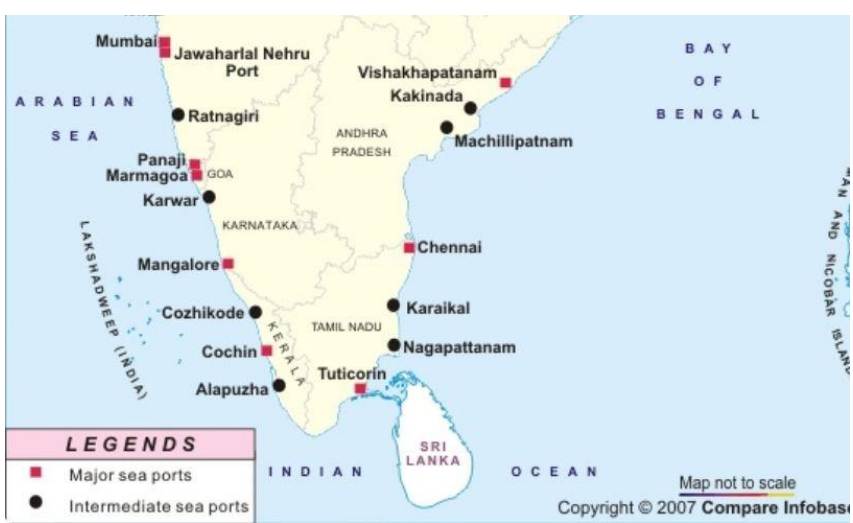

Fig 3. Study area (Indian coastline)

\subsection{Sentinel-1 Data}

This project focuses on the use of Copernicus's Sentinel-1 radar satellite for maritime surveillance. Sentinel-1 satellite is the EU's Copernicus programme constellation and the data acquired by the satellite is freely available on the Copernicus website. The open and free data policy of the Copernicus programme has enabled the organizational, operational, and research work related to radar data more reformed. The availability of such huge amount of SAR imagery encourages for development of new and improved methods in order to process such a large amount of data. Two different regions' Sentinel-1 acquisitions over the Indian water bodies, including coasts and ports, are considered for the analysis of Vessel Detection using SUMO. There are mainly four modes of acquisition of Sentinel-1: Interferometric Wide (IW), Extra Wide (EW), Wave (WV), and Stripmap (SM). Both the employed images are of image mode- IW, which provides a spatial resolution. The acquired data can be prepared for different kinds of the image product, and the Ground Range Detected High resolution (GRDH) product is the one used in this study. Both vertical (V) and horizontal $(\mathrm{H})$ polarization channels can be transmitted by the radar, and either $\mathrm{H}$ or $\mathrm{V}$ or both of them can be received simultaneously. Therefore, a Sentinel-1 acquisition is the subset of the following set combinations: single $-\mathrm{HH}, \mathrm{VV}, \mathrm{HV}$ or $\mathrm{VH}$ and dual $-\mathrm{HV}+\mathrm{HH}$ or $\mathrm{VH}+\mathrm{VV}$, the first and second letter denotes the transmitted and received polarization respectively.

\subsection{Dataset}

The data selected for implementing SUMO consists of the coastline of India. The two Sentinel-1 images are of the Vishakhapatnam port to Hope Island, located on the Bay of Bengal marking the east coast, and the Jawaharlal Nehru Port (also known as Nhava Sheva), located east of Mumbai on the Arabian Sea, marking the west coast. The images of the Sentinel-1 satellite are acquired during May and June, 2018.

SUMO works on images in natural coordinates, range and azimuth. Any type of pre-processing such as orthorectification, filtration or calibration is not needed. It is believed that the pre-processing may result in detection improvements for some type of ships, but the unfiltered radar images are employed and have provided optimal results. The detailed overview of the two Sentinel-1 acquisitions is given below: 
- S1A IW GRDH 1SDV 20180519T002210 20180519T002235 021966 025F4B C9D9

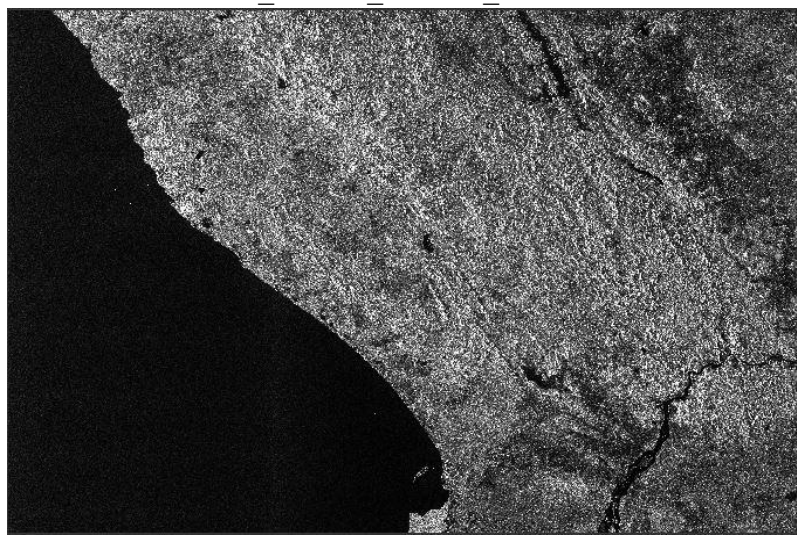

Image

Sensor

Product

Mode

Polarization

Start Time

Duration

Pass

Looking Direction

Size

Mission datatakeid

Instrument

Area of Interest

Main Ports

\author{
20180519T002210 \\ Sentinel-1 \\ GRDH \\ IW \\ $\mathrm{VV} / \mathrm{VH}$ \\ 2018-05-19 04:25:15 \\ $0.698 \mathrm{sec}$ \\ Descending \\ Right \\ $1.59 \mathrm{~GB}$ \\ : 155467 \\ SAR-C \\ Eastern Coast of India \\ Vishakhapatnam Port, \\ Kakinada Port
}

- S1A IW GRDH 1SDV 20180625T010251 20180625T010316 022506 027005 96C5

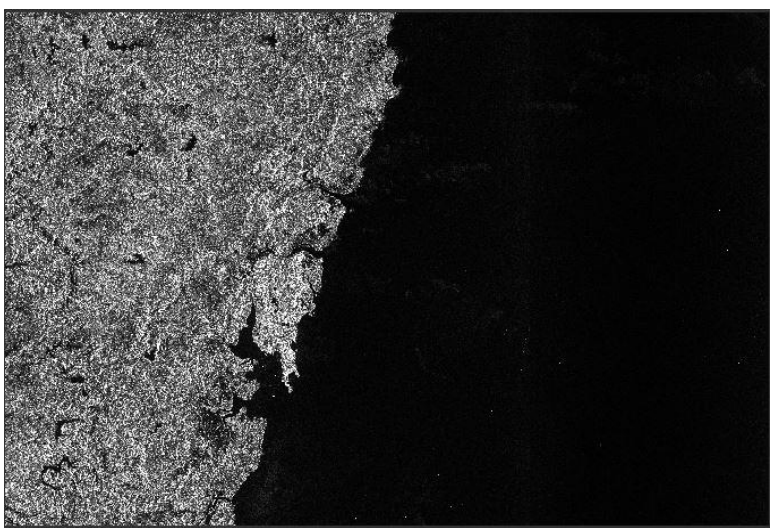

$\begin{array}{ll}\text { Image } & : \text { 20180625T010251 } \\ \text { Sensor } & : \text { Sentinel-1 } \\ \text { Product } & : \text { GRDH } \\ \text { Mode } & : \text { IW } \\ \text { Polarization } & : \text { VV/VH } \\ \text { Start Time } & : 2018-06-25 \text { 12:28:10 } \\ \text { Duration } & : 1.035 \mathrm{sec} \\ \text { Pass } & : \text { Descending } \\ \text { Looking Direction } & : \text { Right } \\ \text { Size } & : 1.61 \mathrm{~GB} \\ \text { Mission datatake id } & : 159749 \\ \text { Instrument } & : \text { SAR-C } \\ \text { Area of Interest } & : \text { Western Coast of India } \\ \text { Main Ports } & : \text { Jawaharlal Port (Mumbai) }\end{array}$

user must select the land mask with seaward buffering by $0 \mathrm{~m}$, which will keep the coastline close to the shore, selecting the maximum port of Vishakhapatnam. The land mask removes the possibility of detecting ambiguities on the surface by creating a type of partition between the land and sea, but the presence of small islands and areas of reefs can never be fully covered by the global coastline land mask. Because they also reflect radar signals and appear bright, these reefs become a common source of false target detections (Harm Greidanus et al., 2017). SUMO calculates a reliability factor indicating the presence or absence of ambiguities, which is responsible for the elimination of such false detection. The reliability value is computed for each of the detected targets after clustering of pixels using its attributes, like size, significance and the likelihood of being an ambiguity. Additionally, this value is affected if the detected target has an implausible (too high or too low) length or width, considering maximum possible ship dimensions. While studying the ship detection results in the Bay of Bengal near the Hope Island, Andra Pradesh, there are many marine object detections falling into different range of size. In every polarization channel, objects are detected which may or may not be a ship. SUMO takes union over the detection results in all the polarization channel, marking the target as detected ship if it has been detected in any of the polarization channels. For example, in figure 5, there are many objects detected in only VV polarization (blue rectangle) or $\mathrm{VH}$ polarization (green triangle) denoting small ships or boats. The detection which is marked in both the 
polarization appears comparatively brighter than the others, denoting a large size object or a regular size ship.

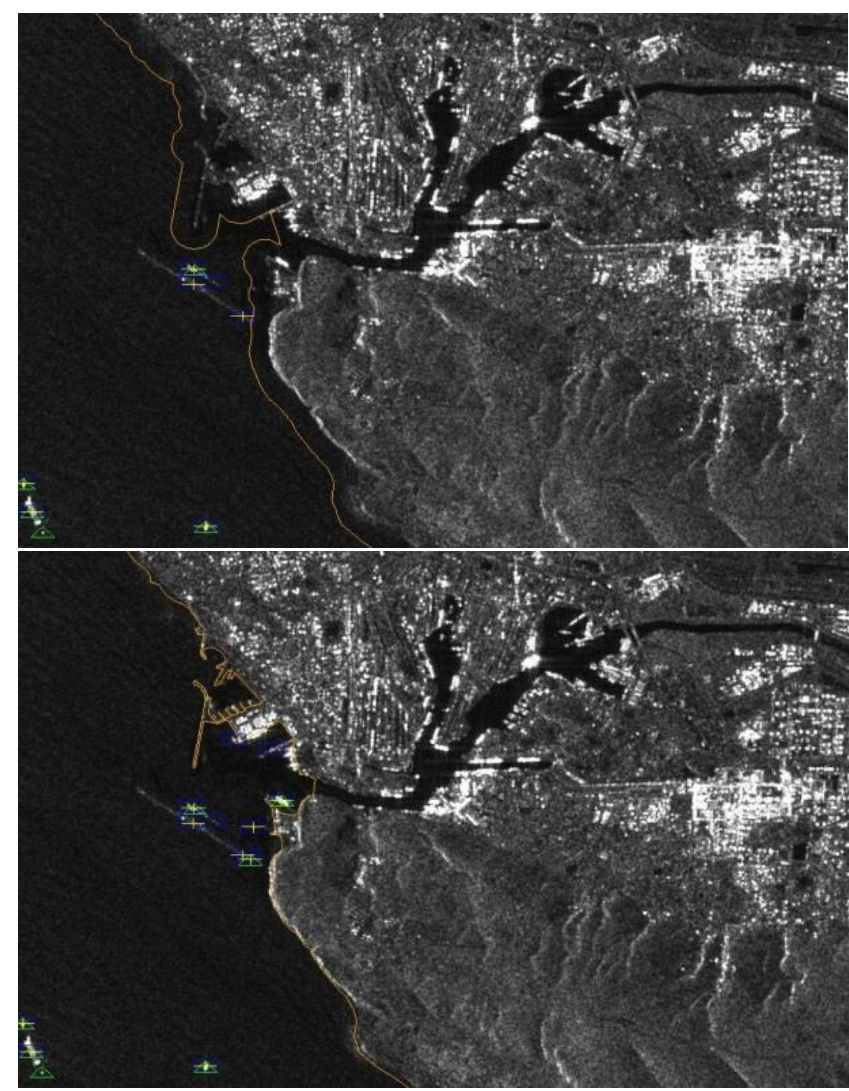

Fig.4 Detection results of SUMO ship detector on the Vishakhapatnam port with different land masks to detect ships in the sea and at the port. Land mask applied are sea buffered by $0 \mathrm{~m}$ (below) and by $250 \mathrm{~m}$ (above).

SUMO was initially developed with the aim of automatic detection of marine objects with minimal human effort but has been improvised with two modes of operation: fully-automatic and semi-automatic. The semi-automatic mode allows the user to manually decide the ship detection result to be discarded or saved. This is particularly helpful if the approximate location of ships is already known in the local area.(H Greidanus et al., 2017) Further, the detection result including the ship's attributes and location can be saved in an output XML file with the information of all detected ships and ambiguities marked by pixels. There are many other output formats supported by SUMO for exporting the detection results such as shapefile (.shp), Google Earth (.kmz), etc. Figure 5 shows the detection result output of a marine object, analyzing its various attributes calculated. Different attributes are saved as the tags in XML denoting a single ship features with single <boat> tag. Besides that, the detection number $<$ target number $>$; geographic location $<$ lon $>,<$ lan $>$; number of pixels which formed the ship $<$ nr_pixels $>$; size $<$ length $>, \quad<$ width $>$; heading direction w.r.t. range $<$ heading range $>$ are some of the main attributes of the detected target. Attributes are a key feature in the detection of any of the automatic identification system as most of the ambiguities can be removed by discarding the detections with attributes having impossible values or not in the range of our desirable output.

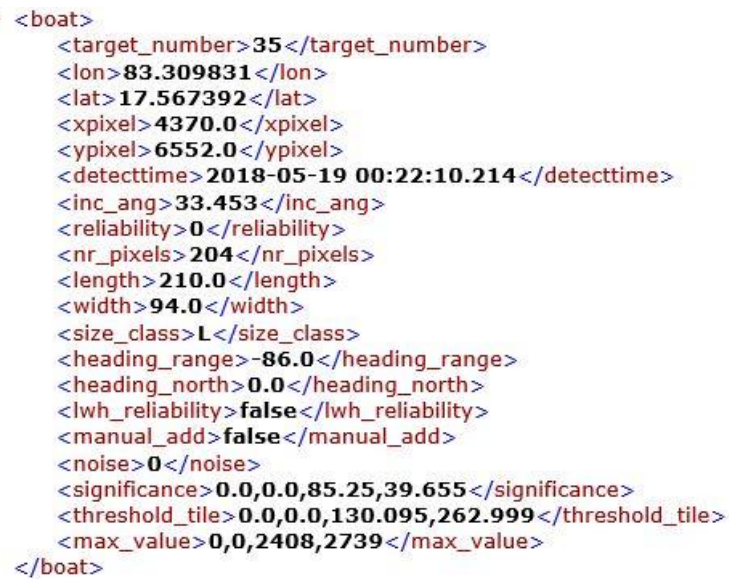

Fig.6 Output result of a ship's attributes in XML format

Table1 depicts the ship detection results of two different Sentinel-1 acquisitions in different regions comparing the total detected ships and ambiguities removed. The two images (discussed in the dataset) are analyzed for the efficiency of SUMO results.

\begin{tabular}{|c|c|c|}
\hline Sensor & Sentinel-1 & Sentinel-1 \\
\hline Area of Interest & $\begin{array}{c}\text { West Coast, } \\
\text { India }\end{array}$ & $\begin{array}{c}\text { East Coast, } \\
\text { India }\end{array}$ \\
\hline Name of Port & $\begin{array}{c}\text { Jawaharlal Port } \\
\text { (Mumbai) }\end{array}$ & $\begin{array}{c}\text { Vishakhapatnam Port, } \\
\text { Kakinada Port (Hope } \\
\text { Island) }\end{array}$ \\
\hline $\begin{array}{c}\text { Product Type- } \\
\text { Image Mode }\end{array}$ & GRDH-IW & GRDH-IW \\
\hline $\begin{array}{c}\text { Date \& Time of } \\
\text { acquisition }\end{array}$ & $\begin{array}{c}2018-06-25, \\
\text { T12:28:10 }\end{array}$ & \begin{tabular}{c} 
T04:32:15 \\
\hline $\begin{array}{c}\text { No. of detected } \\
\text { targets }\end{array}$
\end{tabular} \\
\hline $\begin{array}{c}\text { No. of ships } \\
\text { (detections } \\
\text { with size } \\
\text { class-Large) }\end{array}$ & 1602 & $2018-05-19$, \\
\hline $\begin{array}{c}\text { Ambiguities } \\
\text { Removed }\end{array}$ & 157 & 149 \\
\hline
\end{tabular}

Table-1. SUMO Detection Results 

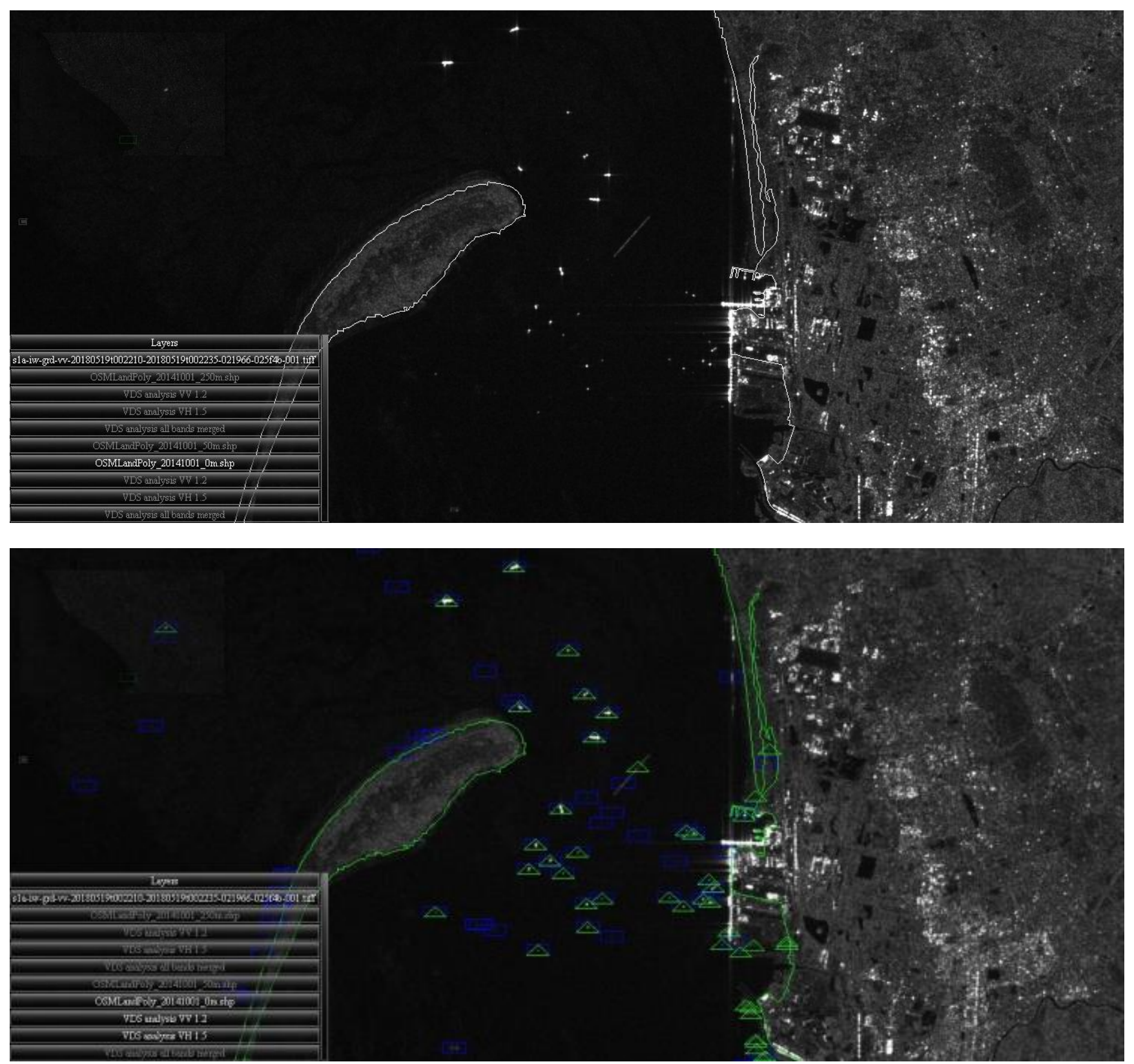

Fig.5 SUMO ship detection results on the port of Kakinada, Hope Island. The image is loaded with the land mask with sea buffering by $0 \mathrm{~m}$ (above) and the detection depicted by different shapes (below). Blue rectangle denotes the detection in VV polarization and green triangle denotes the detection in $\mathrm{VH}$ polarization channel.

\section{Conclusions}

SUMO is a set of algorithms combined for ship detection software which works on the straightforward point detection approach. It is based on the pixel intensities and work on amplitude rather than other measures of SAR. SUMO is a purely CFAR ship detector which provides satisfactory results in the detection of pixels whose values are comparatively higher in their locality. It consolidates an approach with a stochastic model for the sea clutter estimation and is able to eliminate the azimuth ambiguities. There is no attempt to wide range of frequency bands and image modes. It can identify a wide assortment of sea targets of all sizes and shape along with their attributes and features. Featuring fully automatic with a semi automatic mode, SUMO also provides an option for manual recognition and identification. Although there is no doubt that the development of such an algorithm with automatic detection of marine objects with efficient results is an improvement in marine surveillance (using satellite-borne data), there are still many 
approaches which SUMO doesn't explore. It focuses only on the discard the range ambiguities, but has proven to work across a pixel intensities where the detection can be significantly enhanced if the phase or polarization also has been taken into consideration. (Marino, Maria, Hajnsek \& Ouchi ISSN 2072-4292)

$\begin{array}{ll}\text { Abbreviations: } & \\ \text { AIS } & \text { Automatic Identification System } \\ \text { CFAR } & \text { Constant False Alarm Rate } \\ \text { DEM } & \text { Digital Elevation Model } \\ \text { ENL } & \text { Equivalent Number of Looks } \\ \text { ESA } & \text { European Space Agency } \\ \text { EU } & \text { European Union } \\ \text { JRC } & \text { Joint Research Centre } \\ \text { PDF } & \text { Probability Density Function } \\ \text { RCS } & \text { Radar Cross-Section } \\ \text { SAR } & \text { Synthetic Aperture Radar } \\ \text { SLC } & \text { Single-Look Complex } \\ \text { VDS } & \text { Vessel Detection System } \\ \text { VMS } & \text { Vessel Management System }\end{array}$

\section{References}

Harm Greidanus, Marlene Alvarez, Carlos Santamaria, François Xavier Thoorens, Naoum Kourti and Pietro Argentieri: The SUMO Ship Detector Algorithm for Satellite Radar Images, Ispra (VA), Italy 2017 https://www.mdpi.com/2072-4292/9/3/246

Liu, C. A Dual-Polarization Ship Detection Algorithm;Defence Research and Development Canada, Ottawa Research Centre: Ottawa, ON,Canada, 2015.

Vachon, P.W.; Campbell, J.W.M.; Bjerkelund, C.A.; Dobson, F.W.; Rey, M.T. Ship detection by the RADARSAT SAR: Validation of detection model predictions. Can. J. Remote Sens. 1997, 23, 48-59.

Gao, G. A Parzen-Window-Kernel-Based CFAR algorithm for ship detection in SAR Images. IEEE Geosci. Remote Sens. Lett. 2011, 8, 557-561.

Oliver, C.; Quegan, S. Understanding Synthetic Aperture Radar Images; Artech House: Norwood, MA,USA, 1998

Santamaria, C.; Greidanus, H.; Fournier, M.; Eriksen, T.;Vespe, M.; Alvarez, M.; Fernandez Arguedas, V.; Delaney, C.; Argentieri, P. Sentinel-1 contribution to monitoring maritime activity in the arctic.

Wang Juan, Sun Lijie, and Zhang Xuelan Study Evolution of Ship Target Detection and Recognition in SAR Imagery Institute of Technology,Beijing,China 2009

Armando Marino, Maria J. Sanjuan-Ferrer, Iren Hajnsek, and Kazuo Ouchi Ship Detection with Spectral Analysis of Synthetic Aperture Radar: A Comparison of New and Well-Known Algorithms ISSN 2072-4292 www.mdpi.com/journal/remotesensing
Vachon, P.W.; Wolfe, J. GMES Sentinel-1 Analysis of Marine Applications Potential (AMAP); DRDC Ottawa ECR2008-218, Defence Research and Development Canada: Ottawa, ON, Canada, 2008.

Olsen, Richard \& Hannevik, Tonje \& Eldhuset, Knut. (2004). Signatures of vessels in ENVISAT AP-mode imagery. 3895 3897 vol.6. 10.1109/IGARSS.2004.1369975.

Vachon, P.W.; Wolfe, J.; Greidanus, H. Analysis of Sentinel-1 marine applications potential. In Proceedings of the 2012 IEEE International Geoscience and Remote Sensing Symposium, Munich, Germany, 22-27 July 2012

Greidanus, H. Sub-aperture behaviour of SAR signature of ships. In Proceedings of the IGARSS 2006, Denver, CO,USA, 31 July-4 August 2006.

Crisp, D.J. The State-of-the-Art in Ship Detection in Synthetic Aperture Radar Imagery; Defence Science and Technology Organisation, Information Sciences Laboratory: Edinburgh, South Australia, 2004

JRC Technical Reports : The SUMO ship detection software for satellite radar images; H. Greidanus, P. Argentieri, M. Alvarez, C. Santamaria, N. Kourti

JRC Science and Policy Reports on : Sentinel-1 Maritime Surveillance; Carlos Santamaria, Mattia Stasolla, Virginia Fernandez Arguedas, Pietro Argentieri, Marlene Alvarez, Harm Greidanus- 2015.

McCandless, S.W., Jr.; Jackson, C.R. Principles of Synthetic Aperture Radar. In Synthetic Aperture Radar Marine User's Manual; Jackson, C.R., Apel, J.R., Eds.; Department of Commerce, National Oceanic and Atmospheric Administration: Washington, DC, USA, 2004; pp. 1-24.

Pichel, W.G.; Clemente-Colon, P.; Wackerman, C.C.; Friedman, K.S. Ship and wake detection. In Synthetic Aperture Radar Marine User's Manual; Jackson, C.R., Apel, J.R., Eds.; Department of Commerce, National Oceanic and Atmospheric Administration: Washington, DC, USA, 2004; pp. 277-303.

Oliver, C.; Quegan, S. Understanding Synthetic Aperture Radar Images; Artech House: Norwood, MA, USA, 1998.

Greidanus, H. Applicability of the K distribution to RADARSAT maritime imagery. In Proceedings of the IGARSS 2004, Anchorage, AL, USA, 20-24 September 2004; pp. 4715-4718.

Vespe, M.; Greidanus, H. SAR image quality assessment and indicators for vessel and oil spill detection. IEEE Trans. Geosc. Remote Sens. 2012, 50, 4726-4734.

Wackerman, C.C.; Friedman, K.S.; Pichel, W.G.; ClementeColón, P.; Li, X. Automatic detection of ships in RADARSAT-1 SAR imagery. Can. J. Remote Sens. 2001, 27, 371-378. 